\title{
A Study on the Application of Video Assistant Referee (VAR) Technology in Chinese Football Professional League
}

\author{
Jia Zhiliang ${ }^{1, \text { a }}$ \\ ${ }^{1}$ Guangzhou Civil Aviation College, Baiyun Strict, Guangzhou City, Guangdong Province, China
}

\begin{abstract}
By using the methods of literature review, video, video analysis and mathematical statistics, this paper studied the application of VAR technology in 30 rounds and 240 matches in Chinese super league in 2019, and compared it with Super League and 2018 FIFA world cup in 2018. It was found that the accuracy of key penalty was improved, the fairness and justice of the match were guaranteed, and the sound development of Super League was promoted. The application of VAR technology improved the trust between referees and players, promoted the self-confidence of referees in law enforcement and promoted the normal play of players' level. Shortcomings include unreasonable limits and timing of VAR intervention; the understanding and scale of foul penalty are not uniform; the review time is too long and the operation procedure is not standardized, etc. need to be improved.
\end{abstract}

\section{Introduction}

The International Football Council (IFAB) officially launched the test of video assistant referee (VAR) IFAB)2016. After two years of testing, it announced that VAR technology was officially applied to 2018 FIFAWorldCup in March 2018. China Football Professional League started to use VAR technology in Super League in 2018, and started the application of VAR technology in Super League and Chinese League A in 2019, which made China one of the countries that used VAR technology in the highest level and sub-level leagues at the same time. In recent years, Super League has drawn more and more concern. With the development of modern football techniques and tactics, the factors such as the block of matches, fierce confrontation and hidden foul have become the characteristics of the development of modern football. It is more and more difficult for the referee to make a completely correct decision in the competition, and a key decision of the referee in the competition may affect the result of the competition. VAR technology is a very effective supplement to referees in the competition, which can greatly improve the accuracy of referees' decisions, thus ensuring the fairness and justice of the competition. Based on the statistics and analysis of the application data of VAR technology in 30 rounds of competitions in Chinese super league in 2019, this paper explores and ponders the application effect of VAR technology in China's professional football league, aiming at summarizing the rules and finding problems in time, and further improving the practice level of video assistant referee (VAR) technology in China's professional football league, thus promoting the development of China's professional football and playing a positive role in promoting the development of China's professional football league.

\section{Analysis of the overall situation of VAR application in Chinese super league in the 2019 season}

\subsection{Data Analysis of VAR Review Time of Super League in 2019 Season}

TABLE I Analysis of time of review in different levels of leagues using different methods

\begin{tabular}{cccc}
\hline & Total average time & $\begin{array}{c}\text { Average time of on field } \\
\text { reviews }\end{array}$ & $\begin{array}{c}\text { Average time of only VAR } \\
\text { reviews }\end{array}$ \\
\hline 2019 Super League & 81.9 & 86.5 & 55.6 \\
2018 Super League & 114 & 136 & 75 \\
2018 FIFA World Cup & 126 & 131 & 92 \\
\hline
\end{tabular}

The calculation method of elapsed time is from the time when VAR checks the suspected events to the time when the referee makes the final penalty decision. In 2019, the average review analysis time of Super League was 81.9

ae-mail: 121478665@qq.com 
seconds, 86.5 seconds for on-site review analysis and 55.6 seconds for direct review analysis. Looking back and analyzing time is shorter than that of the World Cup in Russia and the Super League in 18 years, which shows that VAR technology has been greatly improved in terms of operation flow, understanding and implementation of VAR protocol after two years of practice.

\subsection{Analysis of the accuracy of penalty after using VAR technology in Super League in 2019 season}

TABLE II Analysis of accuracy rate of penalty decisions in different levels of leagues using different methods.

\begin{tabular}{ccc}
\hline & Without VAR technology & With VAR technology \\
\hline $\begin{array}{c}2019 \text { Super } \\
\text { League }\end{array}$ & $90.3 \%$ & $97.9 \%$ \\
$\begin{array}{c}2018 \text { Super } \\
\text { League }\end{array}$ & $91.3 \%$ & $98.3 \%$ \\
$\begin{array}{c}2018 \text { FIFA World } \\
\text { Cup }\end{array}$ & $96.6 \%$ & $99.35 \%$ \\
\hline
\end{tabular}

In recent years, the Super League has used VAR video technology, which greatly increased the accuracy of penalty in competitions. The accuracy of judgment with VAR technology reached $97.9 \%$ in the 19 th season and $98.3 \%$ in the 18 th season. In the past, the accuracy of penalty in Super League without VAR technology was $90.3 \%$ in the 19 th season and $91.3 \%$ in the 18 th season, which increased by 7 percentage points in both seasons. Both teams, referees, fans and media have played a positive role in the competition, thus providing a fair competition environment and improving the enjoyment of the competition.

\subsection{Super League VAR Review Data Analysis}

In 2019, among the 240 matches in Chinese super league, there were 156 reviews and analyses. Among them, there were 135 on-field reviews and 21 only VAR reviews. The average is 1.53 games. Compared with the 18 -season Super League, the number of on-the-spot reviews increased significantly, while the number of VAR direct reviews decreased significantly.

\begin{tabular}{lccccc} 
TABLE III & $\begin{array}{c}\text { Comparative anaysis ofVAR review data of } 2019 \text { Super League, } 2018 \text { Super } \\
\text { League and } 2018 \text { FIFA World Cup }\end{array}$ \\
\hline & Number of games & Review analysis & On field reviews & $\begin{array}{c}\text { Only VAR } \\
\text { reviews }\end{array}$ & Matches time \\
\hline $\begin{array}{c}2019 \text { China } \\
\text { League One }\end{array}$ & 240 & 156 & 135 & 21 & 1.53 \\
$\begin{array}{c}2018 \text { Super } \\
\text { League }\end{array}$ & 240 & 119 & 75 & 44 & 2.01 \\
$\begin{array}{c}2018 \text { FIFA World } \\
\text { Cup }\end{array}$ & 64 & 20 & 17 & 3 & 3.2 \\
\hline
\end{tabular}

Table VI Comparison of VAR retrospective analysis events in 2018 and 2019

\begin{tabular}{ccccc}
\hline & 2018 season & 2019 season & T value & P value \\
\hline Usage of VAR & $3.97 \pm 1.54$ & $5.20 \pm 2.25$ & -2.33 & 0.03 \\
\hline
\end{tabular}

There is a significant difference between 2018 and 2019 in VAR retrospective analysis events $(\mathrm{P}<0.05)$, indicating that the frequency of use of VAR system increased one year after the introduction of VAR system in 2019.

According to the results of statistical analysis, there are significant differences in VAR in different levels andscenes. No matter what level of competition, there are mistakes and omissions in the key decisions of referees. The higher the level, the less likely the referees will occur, and the less the number of VAR interventions will be. When VAR looked back at the scene, it mainly focused on the fouls that referees need to give subjective punishments, such as penalty kicks and red cards. The direct review of VAR mainly focuses on whether to score offside or not. The referee with the highest level in the World Cup has strong ability, so the proportion of interventions is low, with an average of 3.2 interventions. In 2019, the Chinese Super League had a retrospective analysis every 1.53 games on average, which was more frequently used than the first use of VAR technology in 2018. It was mainly due to the lack of unified understanding of penalty between referees and VAR, and the introduction of $2 \mathrm{D}$ and $3 \mathrm{D}$ line drawing techniques improved the accuracy of VAR in finding offside fouls, which together led to a relatively high proportion of VAR interventions in 2019.

\subsection{Data Analysis of 1-30 rounds of VAR in Super League in 2019}

In 2019, the VAR review data of Super League 30 rounds were arranged irregularly, and the number of reviews was 9 times in the 6th and 14th rounds at most, and 1 time in the 20th round at least. The number of reviews was mostly 5 times. Compared with the 2018 season, its data is more dispersed. 
According to the test statistics, $\mathrm{z}=0.15, \mathrm{P}=0.70>0.05$; It shows that there is no significant linear trend in reviewing the analysis events. According to the schedule arrangement, there should be a correlation between rounds and intensity. However, looking back at the analysis events, there is no linear trend, which shows that the rationality of the use of VAR system is gradually improved with the training mechanism becoming more mature after the second year of its introduction.

TABLE V Analysis of key penalty decision without using VAR in 2019 China League One

\begin{tabular}{ccccccc}
\hline $\begin{array}{c}\text { Test } \\
\text { statistics }\end{array}$ & $\begin{array}{c}\text { Standard } \\
\text { error }\end{array}$ & $\begin{array}{c}\text { Standardized test } \\
\text { statistics }\end{array}$ & $\mathrm{P}$ & $\begin{array}{c}\text { Estimated } \\
\text { value }\end{array}$ & $\begin{array}{c}95 \% \mathrm{CI} \\
\text { lower limit }\end{array}$ & $\begin{array}{c}95 \% \mathrm{CI} \\
\text { upper limit }\end{array}$ \\
\hline 133.00 & 6.25 & 8.73 & 0.00 & 0.85 & 0.79 & 0.90 \\
\hline
\end{tabular}

In the 2019 season, the VAR system of Super League was used 156 times. Among them, 133 times were correct, and VAR system had statistical significance on the correct rate of penalty, $\mathrm{P}<0.01$; The accuracy rate reaches $85.26 \%$, which shows that VAR system can effectively improve the accuracy rate.

\section{Analysis of the specific data of the 2019 VAR Stadium in Chinese super league}

\subsection{Review and analysis of event classification in $\mathbf{3 0}$ rounds of competition in Chinese super league}

TABLE VI Analysis of 156 reviews of four kinds of events in 2019 Super League VAR

\begin{tabular}{ccccc}
\hline Event classification & Number & Percentage & $\begin{array}{c}\text { Changes of penalty } \\
\text { decisions }\end{array}$ & $\begin{array}{c}\text { Affirmation of original } \\
\text { decisions }\end{array}$ \\
\hline Goals & 46 & $29 \%$ & 37 & 9 \\
Red cards & 33 & $21 \%$ & 25 & 8 \\
Penalty kicks & 76 & $48 \%$ & 41 & 0 \\
Wrong object of penalty & 1 & $1 \%$ & 1 & 52 \\
& 156 & $100 \%$ & 104 & \\
\hline
\end{tabular}

There were 156 review events in the 30 rounds of Super League. Among them, 104 referees changed their decision, and 52 referees did not change their decision. Among the four types of retrospective events, the first one was the 76 penalty events, accounting for $48 \%$. The penalty was changed 41 times and unchanged 35 times. In the second place, there were 46 scoring events, accounting for $29 \%$. The penalty was changed 37 times and the penalty was unchanged 9 times. In the third place, there were 33 red card incidents, accounting for $21 \%$. The penalty was changed 25 times and unchanged 8 times. The last one made a mistake, accounting for $1 \%$, and changed the penalty once.

It shows that the focus of the game is always between foul and no foul, whether to score or not and red card events in the penalty area, which are all factors that can directly affect the score and even the result of the game in football match, and the penalty in these key areas often appears wrong judgment, missed judgment and counter-judgment. Therefore, VAR will intervene when the referee makes clear and obvious major mistakes, so as to correct the referee's mistakes and ensure fair competition. However, if VAR is involved in the competition, the referee will change his own view, because VAR is to correct the referee's obvious mistakes. However, when the referee and VAR's views or the penalty scale are not unified, the referee will stick to his own opinion and maintain his original penalty.

\subsection{Analysis of 156 reviews in $\mathbf{3 0}$ rounds of competition in Chinese super league}

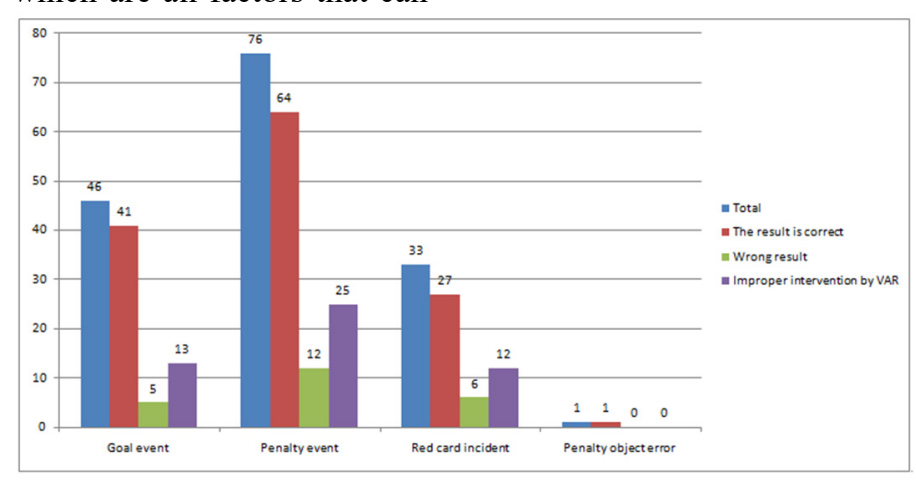

Figure 1 Analysis of VAR 156 penalties in 2019 Super League 
In the practice of 156 reviews of VAR, the results were correct 133 times, wrong 23 times and improper intervention by VAR 60 times. Among them, there were 46 scoring events, 41 correct results, 5 wrong results and 13 inappropriate interventions by VAR. There were 76 penalty events, of which 64 were correct, 12 were wrong and 25 were improperly intervened by VAR. There were 33 red card incidents, of which 27 were correct, 6 were wrong and 12 were improperly intervened by VAR. The penalty object is wrong once, and the result is correct once.

The understanding of scale is not uniform, and the final decision is in the hands of referees, so it is particularly important for referees to understand the rules and identify fouls. This also caused VAR to find clear mistakes or omissions in the match in time, but the referee didn't take VAR's suggestion and insisted on his own point of view, which eventually led to that even if VAR intervened in the match, the final penalty result was wrong. Unclear definition of clear and obvious fouls and omissions leads to many inappropriate interventions of VAR. VAR always finds the referee's omission from the referee's point of view, so as to help the referee check and fill in the gaps, instead of finding the clear and obvious fouls and referees' omissions in four kinds of situations in the game from the perspective of VAR.

\section{Conclusions and suggestions}

\subsection{Conclusion:}

4.1.1 Referees and assistant referees have made good use of delayed whistle blowing and delayed flag drawing techniques, and 2D and 3D line drawing techniques have greatly improved the accurate determination of offside penalty. However, there are also over-reliance on VAR to punish foul and offside, and a lack of foresight and initiative to punish foul.

4.1.2 The application of VAR technology is accurate as a whole, and can ensure efficiency. However, the understanding of VAR agreement is not clear enough, the standards and timing of intervention are not appropriate, and the judgment scale of referees and VAR is not uniform enough. For fouls with different difficulty and degree of difficulty, it takes different time to check and look back. For fouls with high complexity such as penalty kick and direct red card, it takes longer to check and look back.

4.1.3 The application of VAR technology is accurate as a whole, and can ensure efficiency. However, the understanding of VAR agreement is not clear enough, the standards and timing of intervention are not appropriate, and the judgment scale of referees and VAR is not uniform enough. For fouls with different difficulty and degree of difficulty, it takes different time to check and look back. For fouls with high complexity such as penalty kick and direct red card, it takes longer to check and look back.

\subsection{Suggestions}

4.2.1 Fully understand and master the spirit and principles of VAR agreement, strictly abide by the operation process and communication agreement, and standardize the operation. All referees and VAR should unify their thinking, improve their understanding, unite and cooperate, and comply with the requirements of VAR operation process and communication protocol. Strengthen the simulation training of VAR operation, strengthen the awareness of referees and VAR to implement the agreement, and be proficient in the operation process.

4.2.2 Strengthen the inspection and debugging of VAR equipment before and during the competition, enhance the emergency handling ability of VAR equipment problems, make full use of walkie-talkies and PGM (TV broadcast pictures), and play the role of the fourth official as a bridge between VAR and referees. Promote the use of VAR work communication platform as soon as possible, increase the information transmission channels of VAR work, and establish efficient information communication among stadium -VAR operation room-TV broadcast to improve the transparency of VAR work.

\section{ACKNOWLEDGEMENTS}

This research is supported by The project of Guangdong Provincial Bureau of Sports "Research on the Application Status and Prospect of VAR (Video Assistant Referee) Technology in Chinese Professional Football League" Project Number: (GDSS2020N095)

\section{REFERENCES}

1. Lin Jinbiao. The Study on the Features of High Level Football Athletes' Fouls. Sports Sciences Researches. 2016, 20(3): 50-55.

2. $\mathrm{Xu}$ Tao, Liu Zhong. Research on the Dilemma of VAR Technology Application and Its Breakthrough Strategy. Journal of Nanjing Institute of Physical Education: Natural Science.2017, 16(6): 61-65.

3. Zhang Yuhao. Research on the Existing Problems and Countermeasures of VAR Technology Applied in Chinese Professional Football League. Journal of Nanjing Institute of Physical Education. 2018(1): 57-63.

4. $\mathrm{Xu}$ Jinxing, Liu Wei. Research on Video Assistant Referee in Chinese Football Association Super League. Journal of Anhui Sports Science. 2018, 39(1): 19-22. 